\title{
Mathematical Model to Determine Motion of Infinitesimal Mass When Primaries Are both Radiating and Triaxial
}

\author{
${ }^{* 1}$ Atanyi Yusuf Emmanuel., ${ }^{2}$ H.K. Oduwole ${ }^{3}$ Gilbert Aimufua, \\ $\&{ }^{4}$ Abam, Ayeni 0. \\ ${ }^{1,4}$ Department of Mathematics, \\ Federal University of Lafia, \\ PMB 146, Lafia, \\ Nigeria \\ ${ }^{2}$ Department of Mathematics \\ Nasarawa State University, Keffi, \\ PMB 1022 \\ Keffi Nigeria \\ ${ }^{3}$ Department of Computer Science \\ Nasarawa State University, Keffi, \\ PMB 1022 Keffi Nigeria
}

Email: atanyiemmanuel@gmail.com

\begin{abstract}
This model has examined the motion of an infinitesimal particle when both primaries are radiating and triaxial in the framework of the Circular Restricted Three Body Problem (CR3BP). It was observed that the equations of motion were affected by perturbing forces (triaxiality and radiation pressures) of the primary bodies. Analytically, the locations of equilibrium points were obtained and applied to the binary systems Kruger $60(A B)$ and Achird to obtain the numerical results with the help of MathLab software. Numerical investigations reveal that locations of equilibrium points of this problem have been determined and the effect of increasing triaxiality on the position of equilibrium point were obtained with graphical results. It reveal that the infinitesimal mass moves in the direction of the bigger primary towards the line joining the primary bodies. The linear stability of the equilibrium points has also been examined and a numerical solution of the analytical result was applied to the binary stars Kruger 60. It was found that the triangular points are unstable which means that the instability exist as a result of the presence of perturbation forces (Radiation and Triaxiality).
\end{abstract}

Keywords: Model, Motion, Infinitesimal, Radiating, Triaxial.

\section{INTRODUCTION}

Examination of the Restricted Three-Body Problem (R3BP) has been a subject of interest to researchers for over two hundred years and has had significant impact in numerous scientific fields including, among others, celestial mechanics, chaos theory, galactic dynamics and molecular physics. It also finds application in the dynamics of solar and stellar systems, lunar theory and artificial satellites. The R3BP describes profoundly, the motion of three masses in space whose initial positions, velocities and accelerations are 
known leading to the predictions of their future motions. In this motion, the masses are such that they have a common gravitational attraction with the two massive bodies, called primaries, influencing the motion of the third body referred to as, infinitesimal mass having an insignificant effect on the motion of the primaries. Formulation of the restricted threebody problem evolved from the approximate circular motion of planets around the Sun. The satellite of these planets together with small masses of asteroids are compared to the masses of the Sun and the planets. The R3BP constitutes one of the most recognized problems in dynamical astronomy. The exploits witnessed in attempt to understand and explain the dynamics of the R3BP has allowed great historical, theoretical, practical and educational thrive by mankind. The gravitational problem of three bodies in its traditional sense dates in substance from 1687, when Isaac Newton published his "Principia" (Philosophiae Naturalis Principia Mathematic). Newton took the first steps in the definition and study of the problem of the movements of three massive bodies subject to their mutually perturbing gravitational attractions and then applying his results to the lunar theory, the motion of the Moon under the gravitational influence of the Earth and the Sun. However, the accuracy of the lunar theory was low, due to the perturbing effect of the Sun, and planets, on the motion of the Moon around the Earth. The general three-body problem is governed by 18 first order, coupled and non-linear differential equations. Nevertheless, only ten integrals are known to exist and are derived from the conservation of linear momentum, angular momentum, and energy. Hence, these equations of motion do not have a complete analytical solution. Lagrange (1772), in his endeavor to solve the problem, reduced the "three-body problem $(3 B P)$ " to the so-called "restricted three-body problem (R3BP)", in which the moon was assumed to have infinitesimal mass. The substantiating distinction between the general three-body problem and the restricted three-body problem is that primarily, for the latter only two masses are taken arbitrary; with the third mass having an insignificant affection the other two masses. The general three-body problem allows any set of initial conditions for all masses involved; the restricted three-body problem requires the orbit of the masses to be circular or elliptic for which motion is described as either circular or elliptic restricted threebody problem and the motion of the infinitesimal is also required to take place in the orbital plane of the two massive bodies.

The restricted three-body problem was constructed by Lagrange (1772). It was followed by Sharma (1982) and many more modern mathematicians and astronomers which has given rise to the bedrock from which many significant new results have emerged. We can typically see that the man-made satellite has assumed the role of the infinitesimal in the SunEarth/Moon model that was originally developed by Lagrange; with the Earth/Moon emerging as a single entity located at the Earth-Moon barycenter. By including the Earth's non-spherical nature into the problem, Laplace had enhanced the accuracy of the lunar predictions. Ten decades later, when Poynting (1903), began working on the problem, he introduced the idea of a better understanding of the motion of the problem by studying the flow associated with the governing differential equations. Particularly, he tried to describe the motion and stability of three mutually gravitationally attracted bodies. He pointed out a notable fact that any sight variation in initial conditions could amount to a drastically different dynamical behavior. Singh (2007) confirm the first order normalization in the perturbed restricted three body problem (R3BP) with variable masses and the stability of triangular points in the generalized photo gravitational. The classical restricted three-body problem is unable to characterize the motion of the infinitesimal when certain perturbing forces are involved. In recent times, several perturbations such as radiation, variation of masses, tri-axiality, P-R drag etc. have been encompassed in the study of the restricted threebody problem. Poynting had pointed out that perturbations can actually result to variations 
in the locations of the equilibrium points and may change the entire nature of their stability. In this regard, we sought to determine the motion, equilibrium points and linear stability of an infinitesimal mass in the circular restricted three body problem (CR3BP) when both primaries are luminous triaxial rigid bodies.

\section{MATERIALS AND METHODS}

The Lagrangian and Hamiltonian methods are used to derive the equation of motion for the infinitesimal body moving in the vicinity of two massive bodies known as the primaries; orbiting circular path about their common center of mass on account of their mutual gravitational attraction. These primary bodies are such that the bigger primary is radiating, having Poyntin Robertson (P-R) drag effect while the smaller is a triaxial rigid body (Singh \& Amuda 2013; Singh \& Balogun, 2014). Using bary centric rotating reference, the equations of motion are derived in dimensionless parameters. But the formulated model uses the method of second order partial differential equation with the derivations and findings of this work using Matlab R2013a and Mathematica to show the result for the location of triangular equilibrium points with their linear stability.

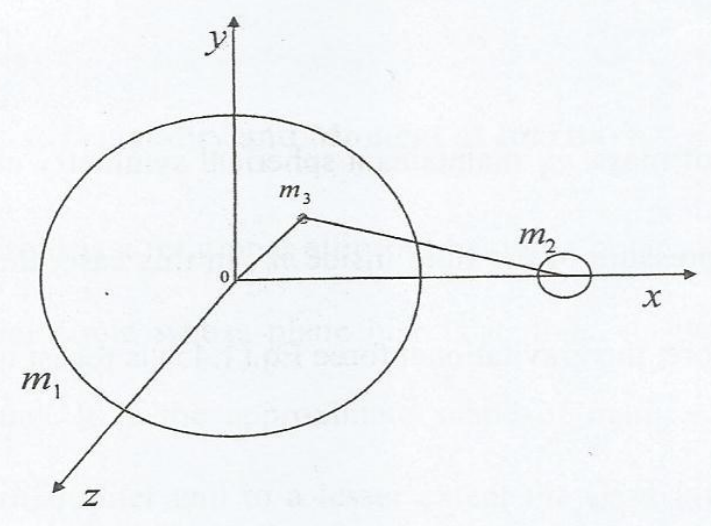

Figure 1: The circular Restricted Three Body Problem

We consider a bary centric coordinate system $O x y z$ which is rotating with respect to an inertial frame of reference and has an angular velocity $\omega$ about their common $z$-axis. The line joining the primaries is taken as the $x$-axis. $m_{1}, m_{2}$ are the masses of the bigger and smaller primaries respectively. It is also assumed that both primaries are radiating and triaxial in nature, with one of the axes taken as the axis of symmetry such that its equatorial plane coincides with the plane of motion. Let $\sigma_{1}, \sigma_{2}$ be triaxial parameters of the bigger primary while $\sigma_{1}, \sigma_{2}$ be those of the smaller primary. We take $(x, y, z)$ as the coordinates of the infinitesimal mass $m$ and its distances from the bigger and smaller primaries are $r_{1}$ and $r_{2}$ respectively. The units of mass, length and time are taken in such a way that the sum of masses of the primaries is unity, the distance between them is unity, and the time period of $m_{2}$ about $m_{1}$ is $2 \pi$ units. Thus the Gaussian gravitational constant $\gamma^{2}=1$. We therefore let the mass parameter be $\mu=\frac{m_{2}}{m_{1}+m_{2}}$ so that $1-\mu=\frac{m_{1}}{m_{1}+m_{2}}$ such that $m_{1}>m_{2}$ and their coordinates are $(-\mu, 0,0)$ and $(1-\mu, 0,0)$ respectively. Then the perturbed mean motion of the primaries is given as $n^{2}=1+\frac{3}{2}\left(2 \sigma_{1}-\sigma_{2}\right)+\frac{3}{2}\left(2 \sigma_{1}^{\prime}-\sigma_{2}^{\prime}\right)$, with $\sigma_{i}, \sigma_{i}^{\prime} \leq 1$ where $\sigma_{1}$ 
$=\left(A_{1}-A_{3}\right) ; \quad \sigma_{2}=\left(A_{2}-A_{3}\right) ; A_{1}=\frac{a^{2}}{5 r_{12}^{2}}, A_{2}=\frac{b^{2}}{5 r_{12}^{2}}, A_{3}=\frac{c^{2}}{5 r_{12}^{2}}$ so that a, b, c are lengths of semi axes of the bigger primary of mass while $\sigma_{1}^{\prime}=\left(A_{1}^{\prime}-A_{3}^{\prime}\right) ; \sigma_{2}^{\prime}=\left(A_{2}^{\prime}-A_{3}^{\prime}\right) ; A_{1}^{\prime}=\frac{a^{\prime 2}}{r_{12}^{\prime 2}}$, $A_{2}^{\prime}=\frac{b^{\prime 2}}{r_{12}^{\prime 2}}, a^{\prime} b^{\prime} c^{\prime}$ are lengths of semi axes of the smaller primary and $r_{12}$ represents the dimensionless distance between the primaries.

The relativistic treatment of the total radiation force emitted by a body was first formulated by Robertson (1937) and later performed by Chernikov (1970). Robertson showed that up to the first order in $\frac{\vec{v}}{c}$, the total sum of radiation force on a given particle say $\mathrm{P}$ due to a radiating body $S$ is

$\vec{F}=\vec{F}_{1}+\vec{F}_{2}+\vec{F}_{3}$

Where $\vec{F}_{1}=F_{p} \frac{\vec{R}}{R}, \vec{F}_{2}=-F_{p} \frac{\vec{v} \cdot \vec{R}}{c R} \frac{\vec{R}}{R}, \vec{F}_{3}=-F_{P} \frac{\vec{v}}{c}$ and $F_{P}=\frac{3 L m}{16 \pi R^{2} \rho s c}$.

$F_{p}$ is the radiation pressure force, $\vec{R}$ stands for the position vector of the particle with respect to the radiation source, $\vec{v}$ is the velocity vector and $c$ is the velocity of light. In the expression of $F_{p}, \mathrm{~L}$ is the luminosity of the radiating body. $m, \rho, s$ Represent the mass, density and cross section of the particle. From equation (1), $F_{1}$ is the radiation pressure, $F_{2}$ is the Doppler shift as a result of the motion of the particle and $F_{3}$ is as a result of absorption and subsequent re-emission of parts of the incident radiation. The forces $F_{2}$ and $F_{3}$ combined are referred to as Poynting-Robertson effect. We also considered the dimensionless velocity of light $\left(c_{d}\right)$ which depends on the masses of the primaries in question and the distance of separation between them.

\section{RESULTS}

In the course of applying the model to understanding the dynamics of stellar bodies; Kruger 60 and Achird, we used Matlab and Mathematica to determine the location of equilibrium point with their graphical solutions and to also ascertain the stability of the equilibrium points. The classical restricted three-body problem is known to admit five equilibrium points, three of which lie along the line joining the primaries, called collinear points $\left(\mathrm{L}_{1}, \mathrm{~L}_{2}\right.$, $\mathrm{L}_{3}$ ), and the other two equilibrium points referred to as the triangular points lie symmetrical to each other above and below the massive bodies. For this study we considered the triangular equilibrium triangular points. Assuming that both primaries are triaxial rigid bodies and are sources of radiation. This means that $\sigma_{i} \neq 0, \sigma_{i}^{\prime} \neq 0 \quad \sigma_{i}, \sigma_{i}^{\prime} \leq 1 i=1,2$. Let $\varepsilon_{1}$ and $\varepsilon_{2}$ represent the perturbations that are caused by the presence of triaxiality, then the values of $r_{1}$ and $r_{2}$ will become

$$
\begin{aligned}
& r_{1}=q_{1}^{\frac{1}{3}}+\varepsilon_{1}, \\
& r_{2}=q_{2}^{\frac{1}{3}}+\varepsilon_{2}, \\
& \left|\varepsilon_{1}\right| \leq 1,\left|\varepsilon_{2}\right| \leq 1
\end{aligned}
$$

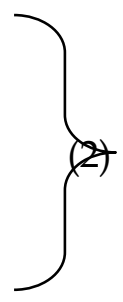


On solving system the exact $x$-coordinate of the triangular liberation point is found to be $x=\frac{1}{2}-\mu+\frac{r_{1}^{2}-r_{2}^{2}}{2}$

Substituting the values of $r_{1}$ and $r_{2}$ from (2) in (3) and ignoring the second and higher order terms of small quantities, we obtain

$$
x=x_{0}+q_{1}^{\frac{1}{3}} \varepsilon_{1}-q_{2}^{\frac{1}{3}} \varepsilon_{2}
$$

With $\quad x_{0}=\frac{1}{2}\left(1-2 \mu+q_{1}^{\frac{2}{3}}-q_{2}^{\frac{2}{3}}\right)$

Again returning to the equation of systems and substituting the value of $x$ using equation of (4) and simplifying, the $y$-coordinate becomes

$y= \pm y_{0}\left(1+\frac{\left(-q_{1}+q_{1}^{\frac{1}{3}} q_{2}^{\frac{2}{3}}+q_{1}^{\frac{1}{3}}\right) \varepsilon_{1}}{2 y_{0}^{2}}+\frac{\left(-q_{2}+q_{1}^{\frac{2}{3}} q_{2}^{\frac{1}{3}}+q_{2}^{\frac{1}{3}}\right) \varepsilon_{2}}{2 y_{0}^{2}}\right)$

Where $y_{0}= \pm \frac{1}{2}\left[2\left\{q_{1}^{\frac{2}{3}}+q_{2}^{\frac{2}{3}}\right\}+2\left\{q_{1} q_{2}\right\}^{\frac{2}{3}}-1-q_{1}^{\frac{4}{3}}-q_{2}^{\frac{4}{3}}\right]^{\frac{1}{2}}$

On substituting $q_{1}=1-\delta_{1}, q_{2}=1-\delta_{2}$ with $\left|\delta_{i}\right| \leq 1,(i=1,2)$ in equations (5) and (7) while neglecting higher order terms of $\varepsilon_{i}, \delta_{i}(i=1,2)$, we have

$$
\begin{aligned}
& x_{0}=\frac{1}{2}-\mu-\frac{\delta_{1}}{3}+\frac{\delta_{2}}{3}, \\
& y_{0}= \pm \frac{\sqrt{3}}{2}\left(1-\frac{2 \delta_{1}}{9}-\frac{2 \delta_{2}}{9}\right)
\end{aligned}
$$

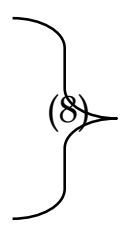

In order to find the values of perturbations $\varepsilon_{1}$ and $\varepsilon_{2}$ we substitute the values $n, x, y, r_{1}$ and $r_{2}$ from (4), (6) and (2) respectively and solving them for $\varepsilon_{1}$ and $\varepsilon_{2}$ while neglecting the second and higher powers of small quantities. Then we have

$$
\begin{aligned}
& \varepsilon_{1}=-\frac{11 \sigma_{1}}{8}+\frac{11 \sigma_{2}}{8}-\frac{(2-3 \mu) \sigma_{1}^{\prime}}{2(1-\mu)}+\frac{(1-2 \mu) \sigma_{2}^{\prime}}{2(1-\mu)}, \\
& \varepsilon_{2}=\frac{(1-3 \mu) \sigma_{1}}{2 \mu}-\frac{(1-2 \mu) \sigma_{2}}{2 \mu}-\frac{11 \sigma_{1}^{\prime}}{8}+\frac{11 \sigma_{2}^{\prime}}{8}
\end{aligned}
$$

On substituting the values of $x_{0}, y_{0}$ and $\varepsilon_{1}, \varepsilon_{2}$ from (8) and (9) respectively, in (3) and (6), then considering only linear terms of small quantities,

We obtain the triangular equilibrium points as:

$$
\begin{aligned}
& x=\frac{1}{2}-\mu-\frac{\delta_{1}}{3}+\frac{\delta_{2}}{3}-\frac{(4+\mu) \sigma_{1}}{8 \mu}+\frac{(4+3 \mu) \sigma_{2}}{8 \mu} \\
& +\frac{(4+3 \mu) \sigma_{1}^{\prime}}{8(1-\mu)}+\frac{(-7+3 \mu) \sigma_{2}^{\prime}}{8(1-\mu)} \\
& y= \pm \frac{\sqrt{3}}{2}\left\{1-\frac{2 \delta_{1}}{9}-\frac{2 \delta_{2}}{9}-\frac{1}{3}\left(\frac{23}{4}-\frac{1}{1-\mu}\right) \sigma_{1}^{\prime}+\frac{1}{3}\left(\frac{19}{4}-\frac{1}{1-\mu}\right) \sigma_{2}^{\prime}\right. \\
& \left.+\frac{1}{3}\left(\frac{-23}{4}+\frac{1}{\mu}\right) \sigma_{1}+\frac{1}{3}\left(\frac{19}{4}-\frac{1}{\mu}\right) \sigma_{2}\right\}
\end{aligned}
$$


Therefore, the points of system (10) and (11) form scalene triangles with the primaries. The positions of these points depend wholly on the mass ratio, triaxiality, radiation pressure. The numerical and graphical results of the analytical results are presented with the help of binary systems and assumed values of some potential in order to obtain the positions of the equilibrium points together with the linear stability of these equilibrium points.

Table 1: Numerical Data

\begin{tabular}{|c|c|c|c|c|c|c|c|c|c|}
\hline \multirow[t]{2}{*}{$\begin{array}{l}\text { Binary } \\
\text { System }\end{array}$} & \multicolumn{2}{|c|}{$\begin{array}{l}\text { Masses } \\
\left(M_{\text {SUN }}\right)\end{array}$} & \multirow[t]{2}{*}{$\begin{array}{l}\text { Mass } \\
\text { ratio }\end{array}$} & \multicolumn{2}{|c|}{$\begin{array}{l}\text { Luminosity } \\
\text { (L LUN) }\end{array}$} & \multicolumn{2}{|c|}{$\begin{array}{l}\text { Radiation } \\
\text { Pressures }\end{array}$} & \multirow{2}{*}{$\begin{array}{l}\text { Binary } \\
\text { Separation } \\
\text { (Asun)A }\end{array}$} & \multirow{2}{*}{$\begin{array}{l}\text { Dimensionless } \\
\text { Velocity } \\
\left(c_{d}\right)\end{array}$} \\
\hline & $\mathrm{M}_{1}$ & $\mathrm{M}_{2}$ & & $\mathrm{~L}_{1}$ & $\mathrm{~L}_{2}$ & $\mathrm{q}_{1}$ & $q_{2}$ & & \\
\hline $\begin{array}{l}\text { Kruger } \\
60 \mathrm{AB})\end{array}$ & 0.271 & 0.176 & 0.3937 & 0.01 & 0.0034 & 0.99992 & 0.99996 & 9.5 & $46,393.84$ \\
\hline Achird & 0.95 & 0.62 & 0.3949 & 1.29 & 0.06 & 0.9971 & 0.9997 & 71 & $67,675.52$ \\
\hline
\end{tabular}

Source: SIMBAD Astronomical Database for the binary stars

Here, we computed numerically the positions of the triangular equilibrium points $\mathrm{L}_{4,5}$ for the binary systems Kruger $60(\mathrm{AB})$ and Achird using equations (10) and (11). The locations of the triangular points for Kruger $60(\mathrm{AB})$ and Achird are presented in tables (2) and (3) respectively. The classical cases $\sigma_{i}=\sigma_{i}^{\prime}=0, q_{i}=1(i=1,2)$ are first shown, then, triaxiality factors of the primary bodies are introduced increasingly before the effects of radiation pressures, triaxiality and the locations of the triangular points of Kruger 60 (AB).

Table 2: Positions of triangular points for Kruger 60 with increasing triaxiality

\begin{tabular}{|c|c|c|c|c|c|c|c|}
\hline $\mathrm{q} 1$ & q2 & o1 & $\sigma 2$ & 03 & $\sigma 4$ & $\mathrm{~L}_{4}$ & $\mathrm{~L}_{5}( \pm)$ \\
\hline 1 & 1 & \multirow[b]{2}{*}{0} & \multirow[b]{2}{*}{0} & \multirow[b]{2}{*}{0} & \multirow[b]{2}{*}{0} & 0.1063 & 0.8660 \\
\hline 0.99992 & 0.99996 & & & & & 0.1063 & 0.8660 \\
\hline 1 & 1 & \multirow[b]{2}{*}{0.0004} & \multirow[b]{2}{*}{0.0003} & \multirow[b]{2}{*}{0.00015} & \multirow[b]{2}{*}{0.00012} & 0.1058 & 0.8658 \\
\hline 0.99992 & 0.99996 & & & & & 0.1057 & 0.8658 \\
\hline 1 & 1 & \multirow[b]{2}{*}{0.004} & \multirow[b]{2}{*}{0.003} & \multirow[b]{2}{*}{0.0015} & \multirow[b]{2}{*}{0.0012} & 0.1009 & 0.8635 \\
\hline 0.99992 & 0.99996 & & & & & 0.1009 & 0.8635 \\
\hline 1 & 1 & \multirow[b]{2}{*}{0.04} & \multirow[b]{2}{*}{0.03} & \multirow[b]{2}{*}{0.015} & \multirow[b]{2}{*}{0.012} & 0.0521 & 0.8411 \\
\hline 0.99992 & 0.99996 & & & & & 0.0521 & 0.8411 \\
\hline 1 & 1 & \multirow[b]{2}{*}{0.4} & \multirow[b]{2}{*}{0.3} & \multirow[b]{2}{*}{0.15} & \multirow[b]{2}{*}{0.12} & -0.4354 & 0.6166 \\
\hline 0.99992 & 0.99996 & & & & & -0.4354 & 0.6166 \\
\hline 1 & 1 & \multirow[b]{2}{*}{0.0004} & \multirow[b]{2}{*}{0.0003} & \multirow[b]{2}{*}{0} & \multirow[b]{2}{*}{0} & 0.1057 & 0.8658 \\
\hline 0.99992 & 0.99996 & & & & & 0.1057 & 0.8658 \\
\hline 1 & 1 & \multirow{3}{*}{0.004} & \multirow[b]{2}{*}{0.003} & \multirow[b]{2}{*}{0} & \multirow[b]{2}{*}{0} & 0.1007 & 0.8642 \\
\hline 0.99992 & 0.99996 & & & & & 0.1007 & 0.8642 \\
\hline 1 & 1 & & \multirow[b]{2}{*}{0.03} & \multirow[b]{2}{*}{0} & \multirow[b]{2}{*}{0} & 0.0505 & 0.8481 \\
\hline 0.99992 & 0.99996 & 0.04 & & & & 0.0505 & 0.8481 \\
\hline 1 & 1 & \multirow[b]{2}{*}{0.4} & & & & -0.4354 & 0.6166 \\
\hline 0.99992 & 0.99996 & & 0.3 & 0 & 0 & -0.4517 & 0.6867 \\
\hline 1 & 1 & & & & & 0.1063 & 0.8660 \\
\hline 0.99992 & 0.99996 & 0 & 0 & 0.0002 & 0.0001 & 0.1063 & 0.8660 \\
\hline 1 & 1 & & & & & 0.1065 & 0.8653 \\
\hline 0.99992 & 0.99996 & 0 & 0 & 0.0015 & 0.0012 & 0.1064 & 0.8653 \\
\hline 1 & 1 & & & & & 0.1079 & 0.8590 \\
\hline 0.99992 & 0.99996 & 0 & 0 & 0.015 & 0.012 & 0.1079 & 0.8590 \\
\hline 1 & 1 & & & & & 0.1226 & 0.7959 \\
\hline 0.99992 & 0.99996 & 0 & 0 & 0.15 & 0.12 & 0.1226 & 0.7958 \\
\hline
\end{tabular}


Table 3: Positions of triangular points for Achird with increasing triaxiality

\begin{tabular}{|c|c|c|c|c|c|c|c|}
\hline $\mathrm{q1}$ & $\mathrm{q} 2$ & o1 & $\sigma 2$ & o3 & $\sigma 4$ & $\mathrm{~L}_{4}$ & $\mathrm{~L}_{5}( \pm)$ \\
\hline 1 & 1 & \multirow[b]{2}{*}{0} & \multirow[b]{2}{*}{0} & \multirow[b]{2}{*}{0} & \multirow[b]{2}{*}{0} & 0.1051 & 0.8660 \\
\hline 0.9971 & 0.9997 & & & & & 0.1042 & 0.8654 \\
\hline 1 & 1 & \multirow[b]{2}{*}{0.0004} & \multirow[b]{2}{*}{0.0003} & \multirow[b]{2}{*}{0.00015} & \multirow[b]{2}{*}{0.00012} & 0.1046 & 0.8658 \\
\hline 0.9971 & 0.9997 & & & & & 0.1032 & 0.8652 \\
\hline 1 & 1 & \multirow[b]{2}{*}{0.004} & \multirow[b]{2}{*}{0.003} & \multirow[b]{2}{*}{0.0015} & \multirow[b]{2}{*}{0.0012} & 0.0997 & 0.8635 \\
\hline 0.9971 & 0.9997 & & & & & 0.0988 & 0.8629 \\
\hline 1 & 1 & \multirow[b]{2}{*}{0.04} & \multirow[b]{2}{*}{0.03} & \multirow[b]{2}{*}{0.015} & \multirow[b]{2}{*}{0.012} & 0.0511 & 0.8411 \\
\hline 0.9971 & 0.9997 & & & & & 0.0502 & 0.8404 \\
\hline 1 & 1 & \multirow[b]{2}{*}{0.4} & \multirow[b]{2}{*}{0.3} & \multirow[b]{2}{*}{0.15} & \multirow[b]{2}{*}{0.12} & -0.4349 & 0.6164 \\
\hline 0.9971 & 0.9997 & & & & & -0.4357 & 0.6158 \\
\hline 1 & 1 & \multirow[b]{2}{*}{0.0004} & \multirow[b]{2}{*}{0.0003} & \multirow[b]{2}{*}{0} & \multirow[b]{2}{*}{0} & 0.1045 & 0.8658 \\
\hline 0.9971 & 0.9997 & & & & & 0.1037 & 0.8652 \\
\hline 1 & 1 & \multirow[b]{2}{*}{0.004} & \multirow[b]{2}{*}{0.003} & \multirow[b]{2}{*}{0} & \multirow[b]{2}{*}{0} & 0.0995 & 0.8642 \\
\hline 0.9971 & 0.9997 & & & & & 0.0987 & 0.8636 \\
\hline 1 & 1 & & \multirow[b]{2}{*}{0.03} & \multirow[b]{2}{*}{0} & \multirow[b]{2}{*}{0} & 0.0495 & 0.8481 \\
\hline 0.9971 & 0.9997 & 0.04 & & & & 0.0486 & 0.8475 \\
\hline 1 & 1 & \multirow[b]{2}{*}{0.4} & & & & -0.4514 & 0.6865 \\
\hline 0.9971 & 0.9997 & & 0.3 & 0 & 0 & -0.4522 & 0.6859 \\
\hline 1 & 1 & & & & & 0.1051 & 0.8660 \\
\hline 0.9971 & 0.9997 & 0 & 0 & 0.0002 & 0.0001 & 0.1042 & 0.8653 \\
\hline 1 & 1 & & & & & 0.1053 & 0.8653 \\
\hline 0.9971 & 0.9997 & 0 & 0 & 0.0015 & 0.0012 & 0.1044 & 0.8647 \\
\hline 1 & 1 & & & & & 0.1067 & 0.8590 \\
\hline 0.9971 & 0.9997 & 0 & 0 & 0.015 & 0.012 & 0.1059 & 0.8584 \\
\hline 1 & 1 & & & & & 0.1216 & 0.7959 \\
\hline 0.9971 & 0.9997 & 0 & 0 & 0.15 & 0.12 & 0.1207 & 0.7953 \\
\hline
\end{tabular}

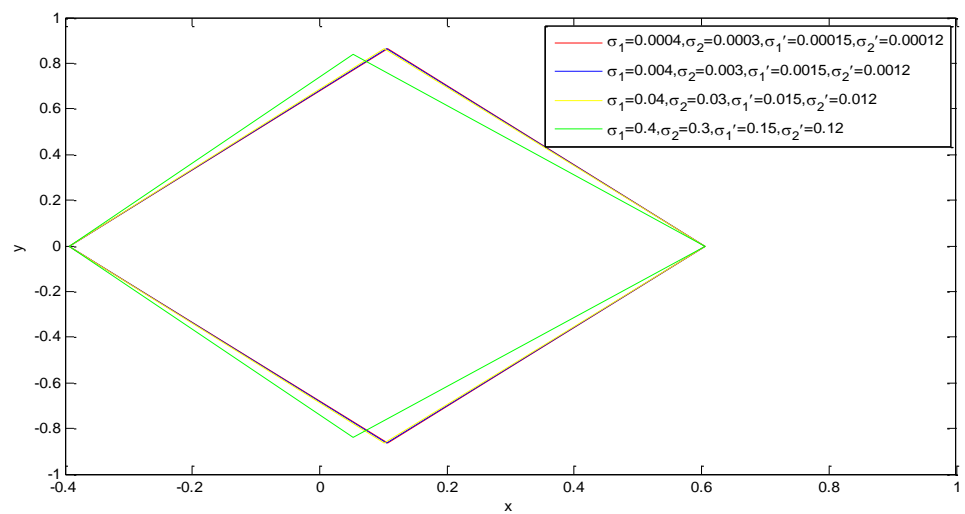

Figure 2: Effect of increasing triaxiality on the position of equilibrium points when both bodies are triaxial For Kruger 60 when $\mathrm{q}_{1}=0.9999$

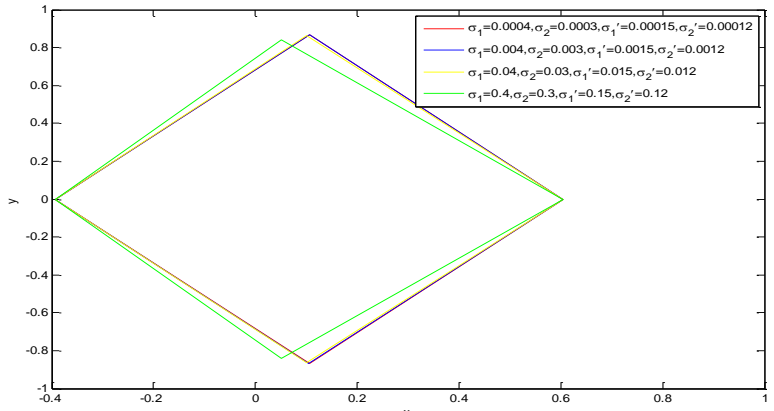

Figure 3: Effect of increasing triaxiality on the position of equilibrium points when both bodies are triaxial for Kruger 60 with $q_{1}=q_{2}=1$ 


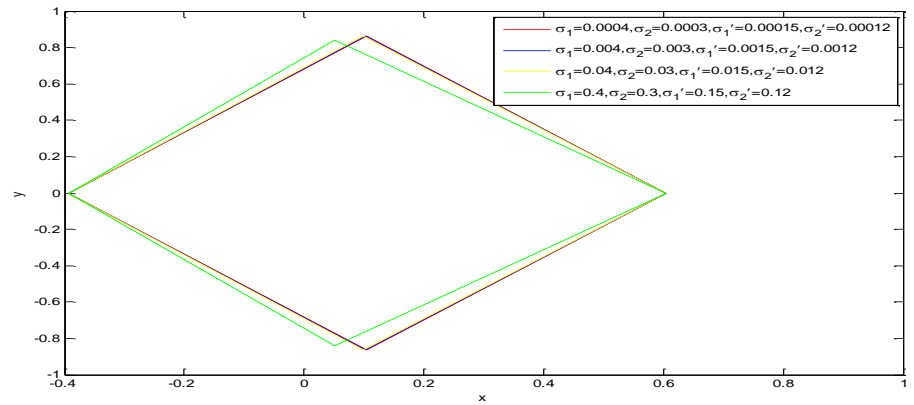

Figure 4: Effect of varying triaxiality on the position of equilibrium points when both bodies are triaxial for Achird 60 with $\mathrm{q}_{1}=0.9971, \mathrm{q}_{2}=0.9997$

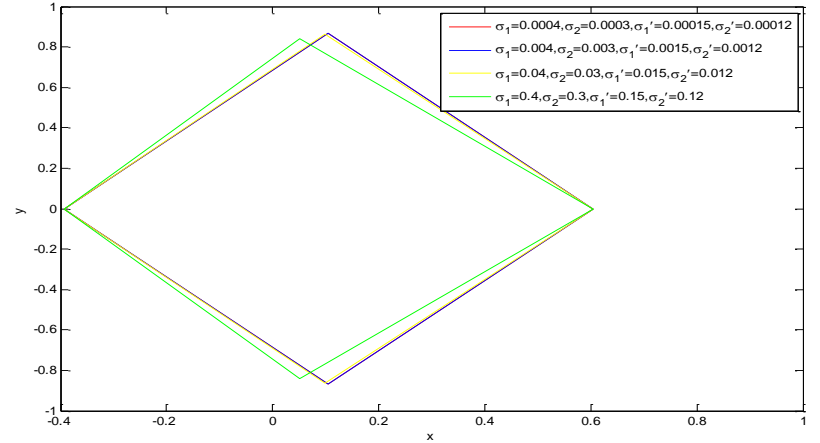

Figure 5: Effect of varying triaxiality on the position of equilibrium points when both bodies are triaxial for Achird 60 with $\mathrm{q}_{1}=1=\mathrm{q}_{2}$

The motion of the infinitesimal mass near one of the equilibrium points is said to be stable if for any given small displacement with small velocity, the body will oscillate for a considerable time period around that point and when the time elapses, it returns to that same point. But when this body departs from the equilibrium point as time increases and does not return to the original point, then the motion of the infinitesimal mass is said to be unstable. To examine the stability of the infinitesimal mass around an equilibrium point, let the coordinates of the equilibrium point be denoted by $\left(x_{0}, y_{0}\right)$ and let $\alpha, \beta$ be small displacements from the point under consideration such that $x=x_{0}+\alpha$ and $y=y_{0}+\beta$, then on substitution we obtain the variational equations of motion as $\ddot{\alpha}-2 n \dot{\beta}=\alpha \Omega_{x x}^{0}+\beta \Omega_{x y}^{0}+\dot{\alpha} \Omega_{x \dot{x}}^{0}+\dot{\beta} \Omega_{x \dot{y}}^{0}$

$\ddot{\beta}+2 n \dot{\alpha}=\beta \Omega_{y y}^{0}+\alpha \Omega_{y x}^{0}+\dot{\alpha} \Omega_{y x}^{0}+\dot{\beta} \Omega_{y \dot{y}}^{0}$

If only linear terms in $\alpha, \beta, \dot{\alpha}, \dot{\beta}$ are taken with the second partial derivatives of $\Omega$ denoted by subscripts while the superscript 0 shows that the derivatives are to be evaluated at the point $\left(x_{0}, y_{0}\right)$. The characteristic equation that corresponds to (12) is written as

$\lambda^{4}+b \lambda^{2}+d=0$

Where

$$
\begin{aligned}
& b=4 n^{2}-\Omega^{o}{ }_{x x}-\Omega^{0}{ }_{y y}+\Omega_{x \dot{x}}^{0} \Omega_{y \dot{y}}^{0}-\left(\Omega_{x \dot{y}}^{0}\right)^{2} \\
& d=\Omega_{x x}^{0} \Omega_{y y}^{0}-\Omega_{x y}^{0} \Omega_{y x}^{0}
\end{aligned}
$$

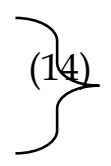


On evaluating the second partial derivatives at the triangular equilibrium point, we have $\Omega^{0}{ }_{x x}=\frac{3}{4}+\delta_{1}\left(\frac{3 \mu}{2}-\frac{1}{2}\right)+\delta_{2}\left(\frac{1}{2}-\frac{3 \mu}{2}\right)+\left(\frac{57}{16}+\frac{45 \mu}{16}-\frac{3}{2 \mu}\right) \sigma_{1}$

$+\left(\frac{3}{16}-\frac{93 \mu}{16}+\frac{3}{2 \mu}\right) \sigma_{2}+\left(\frac{39}{8}-\frac{69 \mu}{16}-\frac{3 \mu^{2}}{2(1-\mu)}\right) \sigma_{1}^{\prime}$

$+\left(\frac{-9}{2}+\frac{117 \mu}{16}+\frac{3 \mu^{2}}{2(1-\mu)}\right) \sigma_{2}^{\prime}$

$\Omega_{y y}^{0}=\frac{9}{4}+\delta_{1}\left(\frac{1}{2}-\frac{3 \mu}{2}\right)+\delta_{2}\left(-1+\frac{3 \mu}{2}\right)+\left(\frac{87}{16}-\frac{45 \mu}{16}+\frac{3}{2 \mu}\right) \sigma_{1}$

$+\left(\frac{-21}{6}+\frac{45 \mu}{16}-\frac{3}{2 \mu}\right) \sigma_{2}+\left(\frac{33}{8}+\frac{135 \mu}{16}-\frac{33 \mu}{8(1-\mu)}+\frac{45 \mu^{2}}{8(1-\mu)}\right) \sigma_{1}^{\prime}$

$+\left(-\frac{135 \mu}{16}+\frac{33 \mu}{8(1-\mu)}-\frac{45 \mu^{2}}{8(1-\mu)}\right) \sigma_{2}^{\prime}$

$\Omega_{x y}=\frac{3 \sqrt{3}}{2}\left\{\frac{1}{2}-\mu-\frac{\delta_{1}(1+\mu)}{9}+\frac{\delta_{2}(2-\mu)}{9}+\left(\frac{47}{24}-\frac{89 \mu}{24}-\frac{1}{3 \mu}\right) \sigma_{1}\right.$

$+\left(\frac{9}{24}-\frac{37 \mu}{24}+\frac{1}{3 \mu}\right) \sigma_{2}+\left(\frac{25}{12}-\frac{85 \mu}{24}+\frac{\mu}{6(1-\mu)}+\frac{\mu^{2}}{6(1-\mu)}\right) \sigma_{1}^{\prime}$

$\left.+\left(\frac{-3}{2}+\frac{11 \mu}{8}-\frac{\mu}{6(1-\mu)}-\frac{\mu^{2}}{6(1-\mu)}\right) \sigma_{2}^{\prime}\right\}$

$\Omega_{y x}=\frac{3 \sqrt{3}}{2}\left\{\frac{1}{2}-\mu-\frac{\delta_{1}(1+\mu)}{9}+\frac{\delta_{2}(2-\mu)}{9}+\left(\frac{47}{24}-\frac{89 \mu}{24}-\frac{1}{3 \mu}\right) \sigma_{1}\right.$

$+\left(\frac{9}{24}-\frac{37 \mu}{24}+\frac{1}{3 \mu}\right) \sigma_{2}+\left(\frac{25}{12}-\frac{85 \mu}{24}+\frac{\mu}{6(1-\mu)}+\frac{\mu^{2}}{6(1-\mu)}\right) \sigma_{1}^{\prime}$

$\left.+\left(\frac{-3}{2}+\frac{11 \mu}{8}-\frac{\mu}{6(1-\mu)}-\frac{\mu^{2}}{6(1-\mu)}\right) \sigma_{2}^{\prime}\right\}$

$\Omega_{x y}^{0}=\Omega_{y x}^{0}$

$\Omega_{x \dot{x}}^{0}=0$

$\Omega_{y \dot{y}}^{0}=0$

$\Omega_{x \dot{y}}^{0}=0$

$\left(\Omega_{x \dot{y}}^{0}\right)^{2}=0$

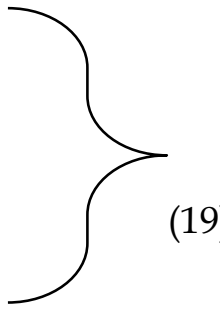

Substituting for the values of $\Omega^{0}{ }_{x x}, \Omega^{0}{ }_{y y}, \Omega^{0}{ }_{x y}, \Omega^{0}{ }_{y x}, \Omega^{0}{ }_{x \dot{x}}, \Omega^{0}{ }_{y \dot{y}}, \Omega^{0}{ }_{x \dot{y}}, \Omega^{0}{ }_{y \dot{x}}$ into equation (16) , we have 


$$
\begin{aligned}
& b=1+\frac{49 \sigma_{1}}{16}+\left(\frac{-43}{16}+3 \mu\right) \sigma_{2} \\
& +\left(3-\frac{33 \mu}{8}+\frac{33 \mu}{8(1-\mu)}-\frac{33 \mu^{2}}{8(1-\mu)}\right) \sigma_{1}^{\prime} \\
& +\left(-\frac{3}{2}+\frac{9 \mu}{8}-\frac{33 \mu}{8(1-\mu)}+\frac{33 \mu^{2}}{8(1-\mu)}\right) \sigma_{2}^{\prime}+\frac{\delta_{2}}{2} \\
& d=\frac{27 \mu(1-\mu)}{4}+\frac{3 \mu(1-\mu) \delta_{1}}{2}+\frac{3}{2}\left(\frac{-3}{4}+\mu-\mu^{2}\right) \delta_{2} \\
& +\left(\frac{-45}{8}+\frac{891 \mu}{16}-\frac{801 \mu^{2}}{16}\right) \sigma_{1}+\left(\frac{-33}{64}+\frac{9 \mu}{2}-\frac{333 \mu^{2}}{16}\right) \sigma_{2} \\
& +\left(\frac{1557 \mu}{32}-\frac{135 \mu}{32(1-\mu)}+\frac{63 \mu^{2}}{32(1-\mu)}-\frac{765 \mu^{2}}{16}+\frac{9 \mu^{3}}{4(1-\mu)}\right) \sigma_{1}^{\prime} \\
& +\left(\frac{-621 \mu}{32}+\frac{135 \mu}{32(1-\mu)}-\frac{63 \mu^{2}}{32(1-\mu)}+\frac{297 \mu^{2}}{16}-\frac{9 \mu^{3}}{4(1-\mu)}\right) \sigma_{2}^{\prime}
\end{aligned}
$$

The general expression for the roots of equation (4.16) are given as

\begin{tabular}{|c|c|c|c|c|c|c|c|}
\hline $\mathrm{q} 1$ & $\mathrm{q} 2$ & o1 & $\sigma 2$ & o3 & o4 & $\lambda_{1,2}$ & $\lambda_{3,4}$ \\
\hline 1 & 1 & \multirow[b]{2}{*}{0} & \multirow[b]{2}{*}{0} & \multirow[b]{2}{*}{0} & \multirow[b]{2}{*}{0} & \pm 1.6112 & \pm 0 \\
\hline 0.9971 & 0.9997 & & & & & \pm 1.6118 & \pm 0 \\
\hline 1 & 1 & \multirow[b]{2}{*}{0.0004} & \multirow[b]{2}{*}{0.0003} & \multirow[b]{2}{*}{0.0002} & \multirow[b]{2}{*}{0.0001} & \pm 1.6137 & \pm 0 \\
\hline 0.9971 & 0.9997 & & & & & \pm 1.6143 & \pm 0 \\
\hline 1 & 1 & & & & & \pm 1.6323 & \pm 0 \\
\hline 0.9971 & 0.9997 & 0.004 & 0.003 & 0.0015 & 0.0012 & \pm 1.6329 & \pm 0 \\
\hline 1 & 1 & 0.04 & 0.03 & 0.015 & 0.012 & \pm 1.8023 & \pm 0 \\
\hline
\end{tabular}

$$
\lambda_{1,2}=-s \pm \frac{1}{2} \sqrt{-4 s^{2}-2 p+\frac{q}{s}} ; \lambda_{3,4}=+s \pm \frac{1}{2} \sqrt{-4 s^{2}-2 p-\frac{q}{s}}
$$

Where

$$
\begin{aligned}
& s=\frac{1}{2} \sqrt{-\frac{2}{3} p+\frac{1}{3}\left(Q+\frac{\Delta_{0}}{Q}\right)} \\
& p=\frac{8 b}{8}, q=\frac{8 c}{8} \\
& Q=\sqrt[3]{\frac{\Delta_{1}+\sqrt{\Delta_{1}^{2}-4 \Delta_{0}^{3}}}{2}} \\
& \Delta_{1}=2 b^{3}-72 b d \\
& \Delta_{0}=b^{2}+12 d
\end{aligned}
$$

Table 4: Roots of characteristic equation for Kruger 60 with varying Triaxiality 


\begin{tabular}{|c|c|c|c|c|c|c|c|}
\hline 0.9971 & 0.9997 & & & & & \pm 1.8028 & \pm 0 \\
\hline 1 & 1 & \multirow[b]{2}{*}{0.4} & \multirow[b]{2}{*}{0.3} & \multirow[b]{2}{*}{0.15} & \multirow[b]{2}{*}{0.12} & \pm 2.6004 & \pm 0 \\
\hline 0.9971 & 0.9997 & & & & & \pm 2.6006 & \pm 0 \\
\hline 1 & 1 & \multirow[b]{2}{*}{0.0004} & \multirow[b]{2}{*}{0.0003} & \multirow[b]{2}{*}{0} & \multirow[b]{2}{*}{0} & \pm 1.6128 & \pm 0 \\
\hline 0.9971 & 0.9997 & & & & & \pm 1.6134 & \pm 0 \\
\hline 1 & 1 & \multirow[b]{2}{*}{0.004} & \multirow[b]{2}{*}{0.003} & \multirow[b]{2}{*}{0} & \multirow[b]{2}{*}{0} & \pm 1.6269 & \pm 0 \\
\hline 0.9971 & 0.9997 & & & & & \pm 1.6275 & \pm 0 \\
\hline 1 & 1 & & \multirow[b]{2}{*}{0.03} & \multirow[b]{2}{*}{0} & \multirow[b]{2}{*}{0} & \pm 1.7578 & \pm 0 \\
\hline 0.9971 & 0.9997 & \begin{tabular}{|l|l|}
0.04 \\
\end{tabular} & & & & \pm 1.7583 & \pm 0 \\
\hline 1 & 1 & \multirow[b]{2}{*}{0.4} & \multirow[b]{2}{*}{0.3} & \multirow[b]{2}{*}{0} & \multirow[b]{2}{*}{0} & \pm 2.5019 & \pm 0 \\
\hline 0.9971 & 0.9997 & & & & & \pm 2.5021 & \pm 0 \\
\hline
\end{tabular}

\section{DISCUSSION OF RESULTS}

A mathematical model formulated for motions of infinitesimal particle under the influence of luminous-triaxial primaries has been investigated in the framework of the circular restricted three-body problem (CR3BP). The equations of motion given shows the effect of radiation and triaxiality in both primaries, and are seen to be different from that of Singh \& Balogun (2014) with bigger primary radiating, having P-R drag effect while the smaller primary remain triaxial. Table 2, shows the effects of radiation and triaxiality on the locations of the triangular points in CR3BP are investigated. It was observed using Kruger 60 that on increasing the effect of triaxaility, the triangular points are seen moving towards the line joining the primaries in the direction of the bigger primary. The same effect is seen by Achird in Table 3, Moreover, as radiation and triaxiality factors increases, there is a shift towards the bigger primary and towards the line joining the primaries in all binaries. Table 1 , presents the necessary numerical data obtained from SIMBAD Astronomical Database for the binary stars under consideration. Using this data, the radiation pressures $q_{1}$ and $q_{2}$ are computed based on Stefan-Boltzmann's law, where $q=1-\frac{A K L}{a \rho M}$. M and L are the mass and Luminosity of a star while $a$ and $\rho$ are the radius and density of a dust grain from radiation. $\mathrm{K}=1$ is the radiation pressure efficiency of the star; $A=\frac{3}{16 \pi C G}$ represents a constant and in C.G.S. system, $A=2.9838 \times 10^{-5}$ we have also taken $a=2 \times 10^{-2} \mathrm{~cm}^{-3}$ and $\rho=1.4 \mathrm{gcm}^{-3}$ for any given dust grain in the systems. The dimensionless velocity of light for the stars is computed as $A=\frac{c}{\sqrt{\frac{\gamma\left(M_{1}+M_{2}\right)}{A_{u}}}}$ Where c, $\mathrm{A}_{\mathrm{u}}$ and $\gamma$ represent the velocity of light, binary separation of the primaries and gravitational constant; $\mathrm{M}_{1}, \mathrm{M}_{2}$ are the masses of the primaries. In C.G.S. system, $\quad c=2.99792458 \times 10^{10} \mathrm{cms}^{-1}$ and $\gamma=6.6743 \times 10^{-8} \mathrm{~cm}^{2} \mathrm{~g}^{-1} \mathrm{~s}^{-2}$.

Numerical experiments were carried out using parameter values in Tables 2 and 3 respectively and applying the commands for the location of triangular equilibrium points. In Figure 2, effect of increasing triaxiality on the position of triangular equilibrium points with both bodies are radiating and triaxial for Kruger 60 . When $\mathrm{q}_{1}=0.99992$ and $\mathrm{q}_{2}=0.99996$, it shows that the position of the equilibrium point moves towards the line joining the primary bodies in the direction of the bigger primary. In Figure 3, effect of increasing triaxiality on 
the position of triangular equilibrium points when both bodies are radiating and triaxial for Kruger 60 with $\mathrm{q}_{1}=\mathrm{q}_{2}=1$. It shows that, the position of the equilibrium point moves towards the line joining the primary bodies in the direction of the bigger primary. In Figure 4, effect of increasing triaxiality on the position of triangular equilibrium points with varying radiation parameter when both bodies are radiating and triaxial for Achird with $\mathrm{q}_{1}=0.9971$, $\mathrm{q}_{2}=0.9997$, also reveals that the position of the equilibrium point moves towards the line joining the primary bodies in the direction of the bigger primary. In Figure 5, effect of increasing triaxiality on the position of triangular equilibrium points with varying radiation parameter when both bodies are radiating and triaxial for Achird with $\mathrm{q}_{1}=\mathrm{q}_{2}=1$, depicts the position of the equilibrium point moving towards the line joining the primary bodies in the direction of the bigger primary. It is obvious that these perturbations reduce the range of stability as seen in Table 4. However, in the absence of perturbations, when $\sigma_{i}=\sigma_{i}^{\prime}=0, q_{i}=1$ with $W_{i}=0(i=1,2)$, the result reduces to the classical problem as contained in Szebehely (1967). But in the absence of triaxiality on the bigger primary, radiation factor on the smaller primary and P-R drag effect on the smaller primary that is when $\sigma_{i}=0, q_{2}=0$ with $W_{2}=0$, the result agrees with Sharma et al. (2001), Singh \&Balogun (2014). In this research, we considered $\sigma_{i} \neq \sigma_{i}^{\prime} \neq 0$, and $q_{i} \neq 1$, the findings reveals that both bodies are radiating and triaxial in nature. Finally, for the stability of the dynamical system, as shown in Table 4, that root of characteristics equation for Kruger 60 with varying triaxiality and radiation, with the condition of stability of linear system stated above as if the roots are real and all negative, the solution is stable. However, if any of the roots are positive, the point is unstable. It was clearly observed base on the results we obtained that, the stability of the equilibrium points is said to be unstable due to the nature of the roots of characteristic equations.

\section{CONCLUSION}

In the CR3BP with luminous-triaxial primaries, our examination reveals that the triangular, equilibrium points are unstable due to the nature of perturbations (Radiation and Triaxiality), on both the bigger and smaller primary. The characteristic equations were observed to be real containing positive roots as computed in Table 4.

\section{REFERENCES}

Chernikov, Yu.A. (1970). The Photo Gravitational Restricted Three Body Problem. Soviet Astronomy AJ. 14(1), 217-223.

Lagrange, J. L. (1772). Theoria Motuum Lunae, Typis Academiae imperialism Scientiarum petropoli. In: Opera Omnia, Series 2, ed. L. Courvoisier, 22.

Poynting, J. H. (1903). Philosophical Transaction of the Royal Society of London Series, A Containing Papers of a Mathematical or Physical Character. Vol 202, 525-552.

Robertson, H.P., (1937). Dynamical effect of radiation in the solar system. Monthly Notice of Royal Astronomy Society, 423-438.

Sharma, R. K., Taqvi, Z. A. and Bhatnagar, K. B. (2001) Existence and Stability of Libration Points in the Restricted Three-Body Problem When the Primaries Are Triaxial Rigid Bodies. Celestial Mechanics and Dynamical Astronomy, 79, 119-133.

Sharma, R.K., (1982). Linear stability of triangular points in the generalized photo-gravitational restricted three-body problem of three bodies in the sun and planetary system. (Edited by Fricke, W., and Teleki, G), Dordrecht: Riedel, 435. 
Singh, J., (2007). Stability of the triangular equilibrium point in the restricted three-body problem with variable mass. Journal of the Nigerian Association of Mathematical Physics, Vol. 11, 287-294.

Singh, J., Amuda, T.O. (2013). Poynting -Robertson (P-R) Drag and Oblateness Effects on Motion Around the Triangular Equilibrium Points in the Photo Gravitational R3BP.Astrophysics and Space Science, 348(2).

Singh, J., Balogun, A. (2014). Stability of Triangular Points in the Photo Gravitational CR3BP with Poynting-Robertson Drag and a Smaller Triaxial Primary. Astrophysics and Space Science, 352(1).

Szebehely, V. (1967). Theory of Orbits. The Restricted Problem of Three-bodies. Academic Press, New York, 222, 301. 